\title{
Research Article Generalized Fractional Master Equation for Self-Similar Stochastic Processes Modelling Anomalous Diffusion
}

\author{
Gianni Pagnini, ${ }^{1}$ Antonio Mura, ${ }^{2}$ and Francesco Mainardi ${ }^{3}$ \\ ${ }^{1}$ CRS4, Centro Ricerche Studi Superiori e Sviluppo in Sardegna, Polaris Building 1, \\ 09010 Pula (CA), Italy \\ ${ }^{2}$ CRESME Research S.p.A., Viale Gorizia 25C, 00199 Roma, Italy \\ ${ }^{3}$ Department of Physics, University of Bologna and INFN, Via Irnerio 46, 40126 Bologna, Italy \\ Correspondence should be addressed to Francesco Mainardi, mainardi@bo.infn.it
}

Received 31 May 2012; Accepted 11 September 2012

Academic Editor: Ciprian A. Tudor

Copyright (C 2012 Gianni Pagnini et al. This is an open access article distributed under the Creative Commons Attribution License, which permits unrestricted use, distribution, and reproduction in any medium, provided the original work is properly cited.

The Master Equation approach to model anomalous diffusion is considered. Anomalous diffusion in complex media can be described as the result of a superposition mechanism reflecting inhomogeneity and nonstationarity properties of the medium. For instance, when this superposition is applied to the time-fractional diffusion process, the resulting Master Equation emerges to be the governing equation of the Erdélyi-Kober fractional diffusion, that describes the evolution of the marginal distribution of the so-called generalized grey Brownian motion. This motion is a parametric class of stochastic processes that provides models for both fast and slow anomalous diffusion: it is made up of self-similar processes with stationary increments and depends on two real parameters. The class includes the fractional Brownian motion, the timefractional diffusion stochastic processes, and the standard Brownian motion. In this framework, the M-Wright function (known also as Mainardi function) emerges as a natural generalization of the Gaussian distribution, recovering the same key role of the Gaussian density for the standard and the fractional Brownian motion.

\section{Introduction}

Statistical description of diffusive processes can be performed both at the microscopic and at the macroscopic levels. The microscopic-level description concerns the simulation of the particle trajectories by opportune stochastic models. Instead, the macroscopic-level description requires the derivation of the evolution equation of the probability density function of the particle displacement (i.e., the Master Equation), which is, indeed, connected 
to the microscopic trajectories. The problem of microscopic and macroscopic descriptions of physical systems and their connection is addressed and discussed in a number of cases by Balescu [1].

The most common examples of this microscopic-to-macroscopic dualism are the Brownian motion process together with the standard diffusion equation and the OrnsteinUhlenbeck stochastic process with the Fokker-Planck equation (see, e.g., [2,3]). But the same coupling occurs for several applications of the random walk method at the microscopic level and the resulting macroscopic description provided by the Master Equation for the probability density function [4].

In many diffusive phenomena, the classical flux-gradient relationship does not hold. In these cases anomalous diffusion arises because of the presence of nonlocal and memory effects. In particular, the variance of the spreading particles does no longer grow linearly in time. Anomalous diffusion is referred to as fast diffusion, when the variance grows according to a power law with exponent greater than 1, and is referred to as slow diffusion, when that exponent is lower than 1 . It is well known that a useful mathematical tool for the macroscopic investigation and description of anomalous diffusion is based on Fractional Calculus [5, 6].

A fractional differential approach has been successfully used for modelling purposes in several different disciplines, for example, statistical physics [7], neuroscience [8], economy and finance [9-12], control theory [13], and combustion science [14, 15]. Further applications of the fractional approach are recently introduced and discussed by Tenreiro Machado [16] and Klafter et al. [17].

Moreover, under a physical point of view, when there is no separation of time scale between the microscopic and the macroscopic level of the process, the randomness of the microscopic level is transmitted to the macroscopic level and the correct description of the macroscopic dynamics has to be in terms of the Fractional Calculus for the space variable [18]. On the other side, fractional integro/differential equations in the time variable are related to phenomena with fractal properties [19].

In this paper, the correspondence microscopic-to-macroscopic for anomalous diffusion is considered in the framework of the Fractional Calculus.

Schneider [20,21], making use of the grey noise theory, introduced a class of selfsimilar stochastic processes termed grey Brownian motion. This class provides stochastic models for the slow anomalous diffusion and the corresponding Master Equation turns out to be the time-fractional diffusion equation. This class of self-similar processes has been extended to include stochastic models for both slow and fast anomalous diffusion and it is named generalized grey Brownian motion [22-24]. Moreover, in a macroscopic framework, this larger class of self-similar stochastic processes is characterized by a Master Equation that is a fractional differential equation in the Erdélyi-Kober sense. For this reason, the resulting diffusion process is named Erdélyi-Kober fractional diffusion [25].

The rest of the paper is organized as follows. In Section 2, the Master Equation approach is briefly described and generalized to a non-Markovian framework. Then, a mechanism of superposition is introduced in order to obtain a "time-stretched" generalization of the non-Markovian formulation. In Section 3, the relationship between a Master Equation in terms of the Erdélyi-Kober fractional derivative operator and the generalized grey Brownian motion is highlighted. Finally, in Section 4 conclusions are given. 


\section{The Master Equation Approach}

\subsection{The Master Equation and Its Generalization}

The equation governing the evolution in time of the probability density function (pdf) of particle displacement $P(x ; t)$, where $x \in \mathcal{R}$ is the location and $t \in \mathcal{R}_{0}^{+}$the observation instant, is named Master Equation (ME). The time $t$ has to be interpreted as a parameter such that the normalization condition $\int P(x ; t) d x=1$ holds for any $t$. In this respect, the ME approach describes the system under consideration at the macroscopic level because it is referred to as an ensemble of trajectories rather than a single trajectory.

The most simple and more famous ME is the parabolic diffusion equation which describes the normal diffusion. Normal diffusion, or Gaussian diffusion, is referred to as a Markovian stochastic process whose pdf satisfies the Cauchy problem:

$$
\frac{\partial P(x ; t)}{\partial t}=\Phi \frac{\partial^{2} P(x ; t)}{\partial x^{2}}, \quad P(x ; 0)=P_{0}(x),
$$

where $\boldsymbol{\Phi}>0$ is called diffusion coefficient and has physical dimension $[\Phi]=L^{2} T^{-1}$. The fundamental solution of (2.1), also named Green function, corresponds to the case with initial condition $P(x ; 0)=P_{0}(x)=\delta(x)$ and turns out to be the Gaussian density:

$$
f(x ; t)=\frac{1}{\sqrt{4 \pi \Phi t}} \exp \left\{-\frac{x^{2}}{4 \Phi t}\right\} .
$$

In this case, the distribution variance grows linearly in time, that is, $\left\langle x^{2}\right\rangle=\int_{-\infty}^{+\infty} x^{2} f(x ; t) d x=$ $2 \nexists t$. The Green function represents the propagator that allows to express a general solution through a convolution integral involving the initial condition $P(x ; 0)=P_{0}(x)$, that is,

$$
P(x ; t)=\int_{-\infty}^{+\infty} f(\xi ; t) P_{0}(x-\xi) d \xi
$$

Diffusion equation (2.1) is a special case of the Fokker-Planck equation [2]

$$
\frac{\partial P}{\partial t}=\left[-\frac{\partial}{\partial x} \boldsymbol{\Phi}_{1}(x)+\frac{\partial^{2}}{\partial x^{2}} \boldsymbol{\Phi}_{2}(x)\right] P(x ; t),
$$

where coefficients $\Phi_{1}(x)$ and $\Phi_{2}(x)>0$ are called drift and diffusion coefficients, respectively.

The Fokker-Planck equation, also known as Kolmogorov forward equation, emerges naturally in the context of Markovian stochastic diffusion processes and follows from the more general Chapman-Kolmogorov equation [3], which also describes pure jump processes.

A non-Markovian generalization can be obtained by introducing memory effects, which means, from a mathematical point of view, that the evolution operator on the righthand side of (2.4) depends also on time, that is,

$$
\frac{\partial P}{\partial t}=\int_{0}^{t}\left[\frac{\partial}{\partial x} \Phi_{1}(x, t-\tau)+\frac{\partial^{2}}{\partial x^{2}} \Phi_{2}(x, t-\tau)\right] P(x ; \tau) d \tau .
$$


A straightforward non-Markovian generalization is obtained, for example, by describing a phase-space process $(v, x)$, where $v$ stands for the particle velocity, as in the Kramers equation for the motion of particles with mass $m$ in an external force field $F(x)$, that is,

$$
\frac{\partial P}{\partial t}=\left[-\frac{\partial}{\partial x} v+\frac{\partial}{\partial v}\left(v-\frac{F(x)}{m}\right)+\frac{\partial^{2}}{\partial v^{2}}\right] P(v, x ; t)
$$

In fact, due to the temporal correlation of particle velocity, eliminating the velocity variable in (2.6) gives a non-Markovian generalized ME of the following form [2]:

$$
\frac{\partial P}{\partial t}=\int_{0}^{t} K(x, t-\tau) \frac{\partial^{2}}{\partial x^{2}} P(x ; \tau) d \tau
$$

where the memory kernel $K(x, t)$ may be an integral operator or contain differential operators with respect to $x$, or some other linear operator.

If the memory kernel $K(x, t)$ were the Gel'fand-Shilov function

$$
K(t)=\frac{t_{+}^{-\mu-1}}{\Gamma(-\mu)}, \quad 0<\mu<1,
$$

where the suffix + is just denoting that the function is vanishing for $t<0$, then ME (2.7) would be

$$
\frac{\partial P}{\partial t}=\int_{0_{-}}^{t^{+}} \frac{(t-\tau)^{-\mu-1}}{\Gamma(-\mu)} \frac{\partial^{2}}{\partial x^{2}} P(x ; \tau) d \tau=D_{t}^{\mu} \frac{\partial^{2} P}{\partial x^{2}},
$$

that is, the time-fractional diffusion equation (see, e.g., [17], and references therein). The operator $D_{t}^{\mu}$ is the Riemann-Liouville fractional differential operator of order $\mu$ in its formal definition according to ([26] equation (1.34)) and it is obtained by using the representation of the generalized derivative of order $n$ of the Dirac delta distribution: $\delta^{(n)}(t)=t_{+}^{-n-1} / \Gamma(-n)$, with proper interpretation of the quotient as a limit if $t=0$. It is here reminded that, for a sufficiently well-behaved function $\varphi(t)$, the regularized Riemann-Liouville fractional derivative of noninteger order $\mu \in(n-1, n)$ is

$$
D_{t}^{\mu} \varphi(t)=\frac{d^{n}}{d t^{n}}\left[\frac{1}{\Gamma(n-\mu)} \int_{0}^{t} \frac{\varphi(\tau) d \tau}{(t-\tau)^{\mu+1-n}}\right] .
$$

For any $\mu=n$ nonnegative integer, it is recovered the standard derivative

$$
D_{t}^{\mu} \varphi(t)=\frac{d^{n}}{d t^{n}} \varphi(t)
$$

For more details, the reader is referred to [26]. 


\subsection{A Physical Mechanism for Time-Stretching Generalization}

It is well known that the "stretched" exponential $\exp \left(-t^{\theta}\right)$ with $t>0$ and $0<\theta<1$, being a completely monotone function, can be written as a linear superposition of elementary exponential functions with different time scales $T$. This follow directly from the well-known formula of the Laplace transform of the unilateral extremal stable density $\mathcal{L}_{\theta}^{-\theta}(\xi)$ (see, e.g., [27]), that is,

$$
\int_{0}^{\infty} e^{-t \xi} \mathcal{L}_{\theta}^{-\theta}(\xi) d \xi=e^{-t^{\theta}}, \quad t>0,0<\theta<1
$$

where

$$
\mathcal{L}_{\theta}^{-\theta}(\xi)=\frac{1}{\pi} \sum_{n=1}^{\infty} \frac{(-1)^{n-1}}{n !} \Gamma(1+n \theta) \sin (n \pi \theta) \xi^{-\theta n-1}
$$

Putting $\xi=1 / T$, it follows that

$$
\int_{0}^{\infty} e^{-t / T} \mathcal{L}_{\theta}^{-\theta}\left(\frac{1}{T}\right) \frac{d T}{T^{2}}=e^{-t^{\theta}}, \quad t>0,0<\theta<1,
$$

and $T^{-2} \mathcal{L}_{\theta}^{-\theta}(1 / T)$ is the spectrum of time-scales $T$.

In the framework of diffusion processes, the same superposition mechanism can be considered for the particle pdf. In fact, anomalous diffusion that emerges in complex media can be interpreted as the resulting global effect of particles that along their trajectories have experienced a change in the values of one or more characteristic properties of the crossed medium, as, for instance, different values of the diffusion coefficient, that is, particles diffusing in a medium that is disorderly layered.

This mechanism can explain, for example, the origin of a time-dependent diffusion coefficient. Consider, for instance, the case of a classical Gaussian diffusion (2.1) where different, but time-independent, diffusion coefficients are experienced by the particles. In fact, let $\rho(\Phi, x, t)$ be the spectrum of the values of $\Phi$ concerning an ensemble of Gaussian densities (2.2) which are solutions of (2.1), that is,

$$
f(x ; t, \Phi)=\frac{1}{\sqrt{4 \pi \Phi t}} \exp \left(-\frac{x^{2}}{4 \Phi t}\right)
$$


where the dependence on the diffusion coefficient $\Phi$ is highlighted in the notation, then, taking care about physical dimensions, in analogy with (2.14):

$$
\begin{aligned}
\int f(x ; t, \Phi) \rho(\Phi, x, t) d \Phi & =\frac{1}{\sqrt{4 \pi C_{\alpha}^{1-\alpha / 2} t^{\alpha}}} \exp \left\{-\frac{x^{2}}{4 C_{\alpha}^{1-\alpha / 2} t^{\alpha}}\right\} \\
& =f\left(x ; \frac{C_{\alpha}^{1-\alpha / 2} t^{\alpha}}{\Phi_{0}}, \Phi_{0}\right) \\
& =f_{*}(x ; t),
\end{aligned}
$$

where $0<\alpha<2, \Phi_{0}$ is a reference diffusion coefficient according to notation adopted in (2.15) and

$$
\rho(\boldsymbol{\Phi}, x, t)=\frac{x^{2-4 / \alpha} t^{3 / 2-\alpha / 2}}{\left(4 C_{\alpha}\right)^{1-2 / \alpha} C_{\alpha}^{(1-\alpha / 2) / 2} \Phi^{3 / 2}} \mathcal{L}_{\alpha / 2}^{-\alpha / 2}\left[\frac{x^{2-4 / \alpha} t}{\left(4 C_{\alpha}\right)^{1-2 / \alpha} \boldsymbol{\Phi}}\right]
$$

Hence, the superposition mechanism corresponds to a "time stretching" in the Gaussian distribution of the form $t \rightarrow C_{\alpha}^{1-\alpha / 2} t^{\alpha} / \Phi_{0}$ and the additional parameter $C_{\alpha}$ has dimension: $\left[C_{\alpha}\right]=\left[L^{2} T^{-\alpha}\right]^{1 /(1-\alpha / 2)}$ (see in the appendix the details for the computation of $\rho(\Phi, x, t)$ ). From now on, in order to lighten the notation, it is set that $\Phi_{0}=1$ and $C_{\alpha}=1$.

Note that the Gaussian pdf in (2.16), that now reads

$$
f_{*}(x ; t)=\frac{1}{\sqrt{4 \pi t^{\alpha}}} \exp \left(-\frac{x^{2}}{4 t^{\alpha}}\right)
$$

can be seen as the marginal distribution of a "stretched" Brownian motion $B\left(t^{\alpha}\right)$. Such a process is actually a stochastic Markovian diffusion process and it is easy to understand that the "anomalous" behavior of the variance (i.e., $\left\langle x^{2}\right\rangle=2 t^{\alpha}$ ) comes from the power-like time stretching. However, the Brownian motion stationarity of the increments is lost due to just the nonlinear time scaling. One can preserve the stationarity on the condition to drop the Markovian property. For instance, the pdf given in (2.18) is also the marginal density function of a fractional Brownian motion $B_{H}(t)$ of order $H=\alpha / 2$. Such a process is Gaussian, self-similar, and with stationary increments ( $H$-sssi).

The ME for the time-stretched Gaussian density $f_{*}(x ; t)=f\left(x ; t^{\alpha}\right)$ can easily be obtained starting from (2.1) and it holds

$$
\frac{\partial f\left(x ; t^{\alpha}\right)}{\partial t^{\alpha}}=\frac{1}{\alpha t^{\alpha-1}} \frac{\partial f_{*}(x ; t)}{\partial t}=\frac{\partial^{2} f_{*}(x ; t)}{\partial x^{2}}
$$

that corresponds, indeed, to a Gaussian diffusion with a time-dependent diffusion coefficient equal to $\alpha t^{\alpha-1}$. Finally, the latter equation can be rewritten in an integral form as follows:

$$
f_{*}(x ; t)=f_{* 0}(x)+\int_{0}^{t} \alpha \tau^{\alpha-1} \frac{\partial^{2} f_{*}}{\partial x^{2}} d \tau .
$$


The fractional Brownian motion is emerged to be a good stochastic model for the previous equation, at least because of its mathematical properties, such as the stationarity of the increments. The fractional Brownian motion is known for its memory properties, that, however, come from its non-Markovian covariance structure. A more general formulation of a non-Markovian process involving directly its probability marginal density $P(x ; t, \nexists)$ can be written in the form of (2.7) as follows:

$$
\frac{\partial P}{\partial t}=\Phi \int_{0}^{t} K(x, t-\tau) \frac{\partial^{2} P(x ; \tau)}{\partial x^{2}} d \tau
$$

It is noteworthy to remark that until the memory kernel is not trivial (i.e., $K(x, t)=\delta(t))$ this equation will not in general give Gaussian densities. Assuming in analogy with (2.16), that there exists a general spectrum $\rho_{G}(\Phi, x, t)$ of values of $\Phi$ such that

$$
\int P(x ; t, \Phi) \rho_{G}(\Phi, x, t) d \Phi=P\left(x ; t^{\gamma}\right)=P_{*}(x ; t)
$$

from which it follows that the superposition mechanism generates the time-stretching $t \rightarrow t^{r}$ and, after the change of variables $\tau=\tau^{\prime \gamma}$, the time-stretched ME corresponding to (2.21) and solved by (2.22) is

$$
\begin{aligned}
\frac{\partial P_{*}(x ; t)}{\partial t} & =\gamma t^{\gamma-1} \int_{0}^{t r} K\left(x, t^{\gamma}-\tau^{\prime \gamma}\right) \frac{\partial^{2} P_{*}\left(x ; \tau^{\prime}\right)}{\partial x^{2}} d \tau \prime^{\gamma} \\
& =\gamma t^{\gamma-1} \int_{0}^{t} K\left(x, t^{\gamma}-\tau^{\gamma}\right) \frac{\partial^{2} P_{*}(x ; \tau)}{\partial x^{2}} \gamma \tau^{\gamma-1} d \tau .
\end{aligned}
$$

Finally, the previous formalism can be furthermore generalized assuming that the superposition mechanism generates a "time stretching" described by a smooth and increasing function $g(t)$, with $g(0)=0$. Since $\partial P_{*} / \partial g(t)=(1 /(\partial g / \partial t))\left(\partial P_{*} / \partial t\right),(2.23)$ turns out to be

$$
\frac{\partial P_{*}}{\partial t}=\frac{d g}{d t} \int_{0}^{t} K[x, g(t)-g(\tau)] \frac{\partial^{2} P_{*}}{\partial x^{2}} \frac{d g}{d \tau} d \tau .
$$

Choosing a power memory kernel like (2.8) guarantees that $P_{*}$ is a probability density and that the process is self-similar [28]. Moreover, by choosing the following "time-stretching" function

$$
g(t)=t^{\alpha / \beta}, \quad 0<\alpha<2,0<\beta \leq 1,
$$


equation (2.24) becomes

$$
\begin{aligned}
\frac{\partial P_{*}}{\partial t} & =\frac{\alpha}{\beta} t^{\alpha / \beta-1} \int_{0_{-}}^{t^{+}} \frac{\left(t^{\alpha / \beta}-\tau^{\alpha / \beta}\right)^{-\mu-1}}{\Gamma(-\mu)} \frac{\partial^{2} P_{*}}{\partial x^{2}} \frac{\alpha}{\beta} \tau^{\alpha / \beta-1} d \tau \\
& =\frac{\alpha}{\beta} t^{\alpha / \beta-1} D_{t^{\alpha / \beta}}^{\mu} \frac{\partial^{2} P_{*}}{\partial x^{2}}
\end{aligned}
$$

that, setting $\mu=1-\beta$, corresponds to the stretched time-fractional diffusion equation (see ([29], equation (5.19)).

In terms of the regularized Riemann-Liouville fractional differential operator (2.10),

(2.26) is the ME corresponding to the following integral evolution equation:

$$
P_{*}(x, t)=P_{* 0}(x)+\frac{1}{\Gamma(\beta)} \frac{\alpha}{\beta} \int_{0}^{t} \tau^{\alpha / \beta-1}\left(t^{\alpha / \beta}-\tau^{\alpha / \beta}\right)^{\beta-1} \frac{\partial^{2} P(x, \tau)}{\partial x^{2}} d \tau,
$$

that was originally introduced by Mura [22] and later discussed in a number of papers [23$25,28,29]$.

\section{The Generalized Fractional Master Equation for Self-Similar Processes}

\subsection{The Erdélyi-Kober Fractional Diffusion: The Generalized Grey Brownian Motion}

It is well known that there exists a relationship between the solutions of a certain class of integral equations that are used to model anomalous diffusion and stochastic processes. In this respect, the density function $P_{*}(x ; t)$, which solves $(2.27)$, could be seen as the marginal pdf of the generalized grey Brownian motion (ggBm) [22-25].

The $\mathrm{ggBm}$ is a special class of $H$-sssi processes with Hurst exponent $H=\alpha / 2$, where according to a common terminology, $H$-sssi means $H$-self-similar stationary increments. The ggBm provides non-Markovian stochastic models for anomalous diffusion, of both slow type, when $0<\alpha<1$, and fast type, when $1<\alpha<2$. The ggBm includes some well-known processes so that it defines an interesting general theoretical framework. In fact, the fractional Brownian motion appears for $\beta=1$, the grey Brownian motion, in the sense of Schneider $[20,21]$, corresponds to the choice $0<\alpha=\beta<1$, and finally the standard Brownian motion is recovered by setting $\alpha=\beta=1$. It is noteworthy to remark that only in the particular case of the Brownian motion the stochastic process is Markovian.

Following Pagnini [25], the integral in the non-Markovian kinetic equation (2.27) can be expressed in terms of an Erdélyi-Kober fractional integral operator $I_{\eta}^{\gamma, \mu}$ that, for a sufficiently well-behaved function $\varphi(t)$, is defined as (see [30] equation (1.1.17))

$$
I_{\eta}^{\gamma, \mu} \varphi(t)=\frac{\eta}{\Gamma(\mu)} t^{-\eta(\mu+\gamma)} \int_{0}^{t} \tau^{\eta(\gamma+1)-1}\left(t^{\eta}-\tau^{\eta}\right)^{\mu-1} \varphi(\tau) d \tau,
$$


where $\mu>0, \eta>0$ and $\gamma \in \mathcal{R}$. Hence (2.27) can be rewritten as [14, 15]

$$
P_{*}(x ; t)=P_{* 0}(x)+t^{\alpha}\left[I_{\alpha / \beta}^{0, \beta} \frac{\partial^{2} P_{*}}{\partial x^{2}}\right] .
$$

Since the ggBm serves as a stochastic model for the anomalous diffusion, this leads to define the family of diffusive processes governed by the $\mathrm{ggBm}$ as Erdélyi-Kober fractional diffusion [25].

The ME corresponding to (2.27) is (2.26). But, since in (2.26) it is used the RiemannLiouville fractional differential operator with a stretched time variable, an abuse of notation occurs. Due to the correspondence between (2.27) and (3.2), the correct expression for the ME (2.26) is obtained by introducing the Erdélyi-Kober fractional differential operator $D_{\eta \mid t}^{\gamma, \mu}$ that is defined, for $n-1<\mu \leq n$, as ([30] equation (1.5.19))

$$
D_{\eta \mid t}^{\gamma, \mu} \varphi(t)=\prod_{j=1}^{n}\left(\gamma+j+\frac{1}{\eta} t \frac{d}{d t}\right)\left(I_{\eta}^{\gamma+\mu, n-\mu} \varphi(t)\right)
$$

From definition (2.10), it follows that the Erdélyi-Kober and the Riemann-Liouville fractional derivatives are related through the formula

$$
D_{1 \mid t}^{-\mu, \mu} \varphi(t)=t^{\mu} D_{t}^{\mu} \varphi(t)
$$

A further important property of the Erdélyi-Kober fractional derivative is the reduction to the identity operator when $\mu=0$, that is,

$$
D_{\eta \mid t}^{\gamma, 0} \varphi(t)=\varphi(t)
$$

Recently, the notions of Erdélyi-Kober fractional integrals and derivatives have been further extended by Luchko [31] and by Luchko and Trujillo [32]. Finally, the ME of the ggBm, or Erdélyi-Kober fractional diffusion, is [25]

$$
\frac{\partial P_{*}}{\partial t}=\frac{\alpha}{\beta} t^{\alpha-1} D_{\alpha / \beta \mid t}^{\beta-1,1-\beta} \frac{\partial^{2} P_{*}}{\partial x^{2}}
$$

\subsection{The Green Function of the Generalized Fractional Master Equation as Marginal pdf of the ggBm}

The Green function corresponding to (3.2) and (3.6) is [22-24, 28]

$$
\mathcal{G}(x ; t)=\frac{1}{2} \frac{1}{t^{\alpha / 2}} M_{\beta / 2}\left(\frac{|x|}{t^{\alpha / 2}}\right),
$$


where $M_{\beta / 2}(z)$ is the $M$-Wright function of order $\beta / 2$, also referred to as Mainardi function $[33,34]$. For a generic order $v \in(0,1)$, it was formerly introduced by Mainardi [35] by the series representation

$$
\begin{aligned}
M_{v}(z) & =\sum_{n=0}^{\infty} \frac{(-z)^{n}}{n ! \Gamma[-v n+(1-v)]} \\
& =\frac{1}{\pi} \sum_{n=1}^{\infty} \frac{(-z)^{n-1}}{(n-1) !} \Gamma(v n) \sin (\pi v n) .
\end{aligned}
$$

For further details, the reader is referred to $[29,36,37]$.

The marginal pdf of the ggBm process describes both slow and fast anomalous diffusion. In fact, the distribution variance turns out to be $\left\langle x^{2}\right\rangle=\int_{-\infty}^{+\infty} x^{2} \mathcal{G}(x ; t) d x=(2 / \Gamma(\beta+$ 1)) $t^{\alpha}$ and the resulting process is self-similar with Hurst exponent $H=\alpha / 2$. The variance law is thus consistent with slow diffusion when $0<\alpha<1$ and fast diffusion when $1<\alpha \leq 2$. However, it is noteworthy to be remarked also that a linear variance growing is possible, even with non-Gaussian pdf, when $\beta \neq \alpha=1$ (in this case the increments are uncorrelated but in general not independent). Moreover, a Gaussian pdf with nonlinear variance growing is observed when $\beta=1$ and $\alpha \neq 1$ (i.e., the fractional Brownian motion).

In Figure 1, it is presented a diagram that allows to identify the elements of the ggBm class, referred to as $B_{\alpha, \beta}(t)$. The top region $1<\alpha<2$ corresponds to the domain of fast diffusion with long-range dependence. In this domain, the increments of the process are positively correlated so that the trajectories tend to be more regular (persistent). It should be noted that long-range dependence is associated to a non-Markovian process which exhibits long-memory properties. The horizontal line $\alpha=1$ corresponds to processes with uncorrelated increments, which could model various phenomena of normal diffusion. For $\alpha=\beta=1$, the Gaussian process of the standard Brownian motion is recovered. The Gaussian process of the fractional Brownian motion is identified by the vertical line $\beta=1$. The bottom region $0<\alpha<1$ corresponds to the domain of slow diffusion. The increments of the corresponding process turn out to be negatively correlated and this implies that the trajectories are strongly irregular (antipersistent motion); the increments form a stationary process which does not exhibit long-range dependence. Finally, the lower diagonal line $(\alpha=\beta)$ represents the Schneider grey Brownian motion, whereas the upper diagonal line indicates its "conjugated" process.

It is interesting to observe that the ggBm turns out to be a direct generalization of a Gaussian process not only because it includes Gaussian processes as particular cases, but also because it is possible to show that it can be defined only by giving its autocovariance structure. In other words, the ggBm provides an example of a stochastic process characterized only by the first and second moments, which is indeed a property of Gaussian processes $[23,24]$. Then the $\mathrm{ggBm}$ is a direct generalization of the Gaussian processes and, in the same way, the Mainardi function $M_{v}$ could be seen as a generalization of the Gaussian function, emerging to be the marginal pdf of non-Markovian diffusion processes able to provide models for both slow and fast anomalous diffusion.

Special cases of ME (3.6) are straightforwardly obtained [25]. In particular, it reduces to the time-fractional diffusion if $\alpha=\beta<1$, to the stretched Gaussian diffusion if $\alpha \neq 1$ and $\beta=1$, and finally to the standard Gaussian diffusion if $\alpha=\beta=1$. 


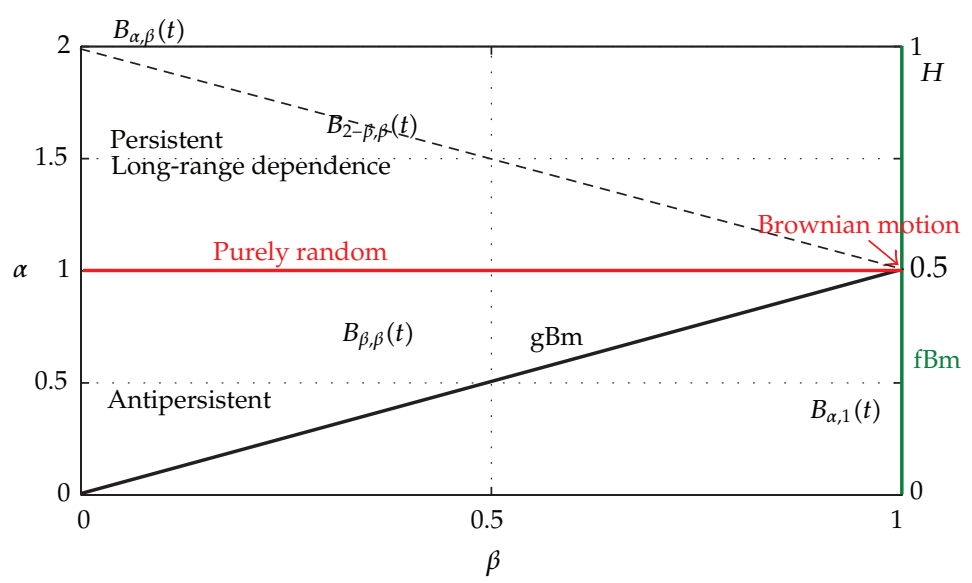

Figure 1: Parametric class of generalized grey Brownian motion.

\section{Conclusions}

In the present paper, the Master Equation approach to model anomalous diffusion of selfsimilar processes has been considered, while generalizations obtained including "timestretching" (or, more generally, time-changing) have been investigated. In particular, it has been shown that such "time stretching" (which could be also obtained through the introduction of time-dependent diffusion coefficients) can physically emerge by the superposition of pdfs concerning the diffusion of particles that along their trajectory have experienced one or more different characteristic of the crossed medium, distributed according to a density spectrum. Then, particles diffuse in a medium that is disorderly layered. In this work, we have considered inhomogeneity and nonstationarity in the spectrum of the diffusion coefficient. This physical idea can be considered as the generation mechanism of anomalous diffusion in complex media.

By the time-stretching of the power law memory kernel within the time-fractional diffusion equation, the $\mathrm{ME}$ of the ggBm follows. The relationship between the valuable family of stochastic processes represented by the ggBm and the Erdélyi-Kober fractional operators is highlighted. In fact, the pdf of particle displacement associated to the $\mathrm{ggBm}$ is the solution of a fractional integral equation (3.2), or, analogously, of a fractional diffusion equation (3.6) in the Erdélyi-Kober sense, and this solution is provided by a transcendental function of the Wright type, also referred to as Mainardi function. Since the governing equation of these processes is a fractional equation in the Erdélyi-Kober sense, it is natural to call this family of diffusive processes as Erdélyi-Kober fractional diffusion.

\section{Appendix}

The inhomogeneous and nonstationary spectrum $\rho(\Phi, x, t)$ of values of $\Phi$ can be computed as follows. Consider formula (2.12) and by introducing a parameter $C_{\alpha}$ and setting $\theta=\alpha / 2$ it turns out to be

$$
\int_{0}^{\infty} e^{-C_{\alpha} s \xi} \mathcal{L}_{\alpha / 2}^{-\alpha / 2}(\xi) d \xi=e^{-C_{\alpha}^{\alpha / 2} s^{\alpha / 2}}, \quad 0<\alpha<2
$$


Appling the change of variables

$$
S=\left(\frac{x^{2}}{4 C_{\alpha} t^{\alpha}}\right)^{2 / \alpha}, \quad \xi=\frac{x^{2-4 / \alpha} t}{\left(4 C_{\alpha}\right)^{1-2 / \alpha} \Phi}
$$

it holds

$$
\int_{0}^{\infty} \exp \left\{-\frac{x^{2}}{4 \Phi t}\right\} \frac{x^{2-4 / \alpha} t}{\left(4 C_{\alpha}\right)^{1-2 / \alpha} \Phi^{2}} \mathcal{L}_{\alpha / 2}^{-\alpha / 2}\left[\frac{x^{2-4 / \alpha} t}{\left(4 C_{\alpha}\right)^{1-2 / \alpha} \Phi}\right] d \Phi=\exp \left\{-\frac{x^{2}}{4 C_{\alpha}^{1-\alpha / 2} t^{\alpha}}\right\}
$$

Finally, dividing both sides by $\sqrt{4 \pi C_{\alpha}^{1-\alpha / 2} t^{\alpha}}$, it results

$$
\begin{aligned}
& \int_{0}^{\infty} \frac{1}{\sqrt{4 \pi \Phi t}} \exp \left\{-\frac{x^{2}}{4 \Phi t}\right\} \times \frac{x^{2-4 / \alpha} t^{3 / 2-\alpha / 2}}{\left(4 C_{\alpha}\right)^{1-2 / \alpha} C_{\alpha}^{(1-\alpha / 2) / 2} \Phi^{3 / 2}} \mathcal{L}_{\alpha / 2}^{-\alpha / 2}\left[\frac{x^{2-4 / \alpha} t}{\left(4 C_{\alpha}\right)^{1-2 / \alpha} \Phi}\right] d \Phi \\
& =\frac{1}{\sqrt{4 \pi C_{\alpha}^{1-\alpha / 2} t^{\alpha}}} \exp \left\{-\frac{x^{2}}{4 C_{\alpha}^{1-\alpha / 2} t^{\alpha}}\right\},
\end{aligned}
$$

from which spectrum (2.17) is recovered.

\section{Acknowledgments}

G. Pagnini is funded by Regione Autonoma della Sardegna (PO Sardegna FSE 2007-2013 sulla L.R. 7/2007 "Promozione della ricerca scientifica e dell'innovazione tecnologica in Sardegna"). Paper presented at FDA12 (Fractional Differentiation and Applications), Nanjing (China) 14-17 May 2012.

\section{References}

[1] R. Balescu, Statistical Dynamics. Matter Out of Equilibrium, Imperial College Press, London, UK, 1997.

[2] H. Risken, The Fokker-Planck Equation: Methods of Solution and Applications, Springer, Berlin, Germany, 2nd edition, 1989.

[3] C. W. Gardiner, Handbook of Stochastic Methods for Physics, Chemistry and the Natural Sciences, Springer, Berlin, Germany, 2nd edition, 1990.

[4] G. H. Weiss, Aspects and Applications of the Random Walk, North-Holland Publishing, Amsterdam, The Netherlands, 1994.

[5] D. Baleanu, K. Diethelm, E. Scalas, and J. J. Trujillo, Fractional Calculus: Models and Numerical Methods, vol. 3 of Series on Complexity, Nonlinearity and Chaos, World Scientific, Singapore, 2012.

[6] K. Diethelm, The Analysis of Fractional Differential Equations, Springer, Berlin, Germany, 2010.

[7] R. Metzler and J. Klafter, "The restaurant at the end of the random walk: recent developments in the description of anomalous transport by fractional dynamics," Journal of Physics A, vol. 37, no. 31, pp. R161-R208, 2004.

[8] B. N. Lundstrom, M. H. Higgs, W. J. Spain, and A. L. Fairhall, "Fractional differentiation by neocortical pyramidal neurons," Nature Neuroscience, vol. 11, no. 11, pp. 1335-1342, 2008.

[9] E. Scalas, R. Gorenflo, and F. Mainardi, "Fractional calculus and continuous-time finance," Physica A, vol. 284, no. 1-4, pp. 376-384, 2000. 
[10] F. Mainardi, M. Raberto, R. Gorenflo, and E. Scalas, "Fractional calculus and continuous-time finance. II: The waiting-time distribution," Physica A, vol. 287, no. 3-4, pp. 468-481, 2000.

[11] R. Gorenflo, F. Mainardi, E. Scalas, and M. Raberto, "Fractional calculus and continuous-time finance. III. The diffusion limit," in Trends in Mathematics-Mathematical Finance, M. Kohlmann and S. Tang, Eds., pp. 171-180, Birkhäuser, Basel, Switzerland, 2001.

[12] E. Scalas, "The application of continuous-time random walks in finance and economics," Physica A, vol. 362, no. 2, pp. 225-239, 2006.

[13] B. M. Vinagre, I. Podlubny, A. Hernández, and V. Feliu, "Some approximations of fractional order operators used in control theory and applications," Fractional Calculus $\mathcal{E}$ Applied Analysis, vol. 3, no. 3, pp. 231-248, 2000.

[14] G. Pagnini, "Nonlinear time-fractional differential equations in combustion science," Fractional Calculus and Applied Analysis, vol. 14, no. 1, pp. 80-93, 2011.

[15] G. Pagnini, "The evolution equation for the radius of a premixed flame ball in fractional diffusive media," in European Physical Journal Special Topics, vol. 193, pp. 105-117, 2011.

[16] J. A. Tenreiro Machado, "And I say to myself: "What a fractional world!"," Fractional Calculus and Applied Analysis, vol. 14, pp. 635-654, 2011.

[17] J. Klafter, S. C. Lim, and R. Metzler, Eds., Fractional Dynamics. Recent Advances, World Scientific, Singapore, 2012.

[18] P. Grigolini, A. Rocco, and B. J. West, "Fractional calculus as a macroscopic manifestation of randomness," Physical Review E, vol. 59, no. 3, pp. 2603-2613, 1999.

[19] A. Rocco and B. J. West, "Fractional calculus and the evolution of fractal phenomena," Physica A, vol. 265, pp. 535-546, 1999.

[20] W. R. Schneider, "Grey noise," in Stochastic Processes, Physics and Geometry, S. Albeverio, G. Casati, U. Cattaneo, D. Merlini, and R. Moresi, Eds., pp. 676-681, World Scientific, Teaneck, NJ, USA, 1990.

[21] W. R. Schneider, "Grey noise," in Ideas and Methods in Mathematical Analysis, Stochastics, and Applications, S. Albeverio, J. E. Fenstad, H. Holden, and T. Lindstrøm, Eds., vol. 1, pp. 261-282, Cambridge University Press, Cambridge, UK, 1992.

[22] A. Mura, Non-markovian stochastic processes and their applications: from anomalous diffusion to time series analysis [Ph.D. thesis], University of Bologna, 2008, Now available by Lambert Academic Publishing 2011.

[23] A. Mura and F. Mainardi, "A class of self-similar stochastic processes with stationary increments to model anomalous diffusion in physics," Integral Transforms and Special Functions, vol. 20, no. 3-4, pp. 185-198, 2009.

[24] A. Mura and G. Pagnini, "Characterizations and simulations of a class of stochastic processes to model anomalous diffusion," Journal of Physics A, vol. 41, no. 28, Article ID 285003, 2008.

[25] G. Pagnini, "Erdélyi-Kober fractional diffusion," Fractional Calculus and Applied Analysis, vol. 15, no. 1 , pp. 117-127, 2012.

[26] R. Gorenflo and F. Mainardi, "Fractional calculus: integral and differential equations of fractional order," in Fractals and Fractional Calculus in Continuum Mechanics, A. Carpinteri and F. Mainardi, Eds., pp. 223-276, Springer, 1997.

[27] F. Mainardi, Y. Luchko, and G. Pagnini, "The fundamental solution of the space-time fractional diffusion equation," Fractional Calculus E Applied Analysis, vol. 4, no. 2, pp. 153-192, 2001.

[28] A. Mura, M. S. Taqqu, and F. Mainardi, "Non-Markovian diffusion equations and processes: analysis and simulations," Physica A, vol. 387, no. 21, pp. 5033-5064, 2008.

[29] F. Mainardi, A. Mura, and G. Pagnini, "The M-Wright function in time-fractional diffusion processes: a tutorial survey," International Journal of Differential Equations, vol. 2010, Article ID 104505, 29 pages, 2010.

[30] V. Kiryakova, Generalized Fractional Calculus and Applications, vol. 301, Longman Scientific \& Technical, Harlow, UK, 1994.

[31] Y. Luchko, "Operational rules for a mixed operator of the Erdélyi-Kober type," Fractional Calculus $\mathcal{E}$ Applied Analysis, vol. 7, no. 3, pp. 339-364, 2004.

[32] Y. Luchko and J. J. Trujillo, "Caputo-type modification of the Erdélyi-Kober fractional derivative," Fractional Calculus \& Applied Analysis, vol. 10, no. 3, pp. 249-267, 2007.

[33] F. Mainardi, Fractional Calculus and Waves in Linear Viscoelasticity, Imperial College Press, London, UK, 2010.

[34] I. Podlubny, Fractional Differential Equations, vol. 198, Academic Press, San Diego, Calif, USA, 1999.

[35] F. Mainardi, "Fractional relaxation-oscillation and fractional diffusion-wave phenomena," Chaos, Solitons and Fractals, vol. 7, no. 9, pp. 1461-1477, 1996. 
[36] R. Gorenflo, Y. Luchko, and F. Mainardi, "Analytical properties and applications of the Wright function," Fractional Calculus \& Applied Analysis, vol. 2, no. 4, pp. 383-414, 1999.

[37] R. Gorenflo, Y. Luchko, and F. Mainardi, "Wright functions as scale-invariant solutions of the diffusion-wave equation," Journal of Computational and Applied Mathematics, vol. 118, no. 1-2, pp. 175$191,2000$. 


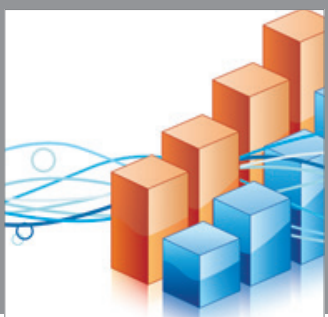

Advances in

Operations Research

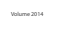

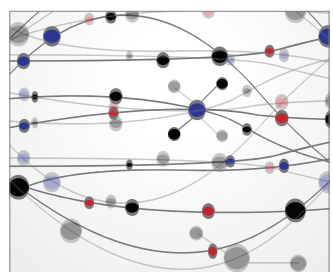

\section{The Scientific} World Journal
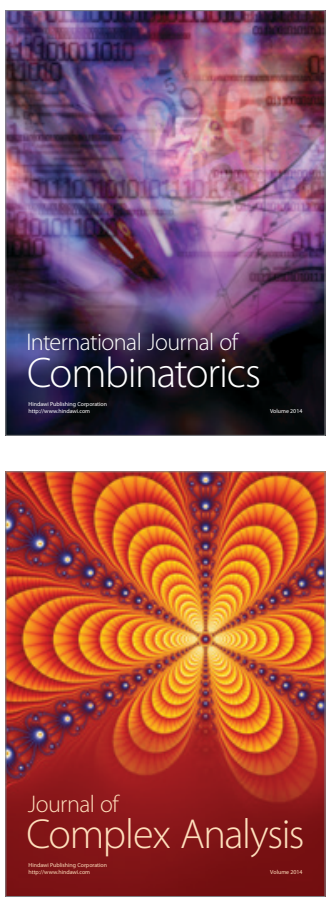

International Journal of

Mathematics and

Mathematical

Sciences
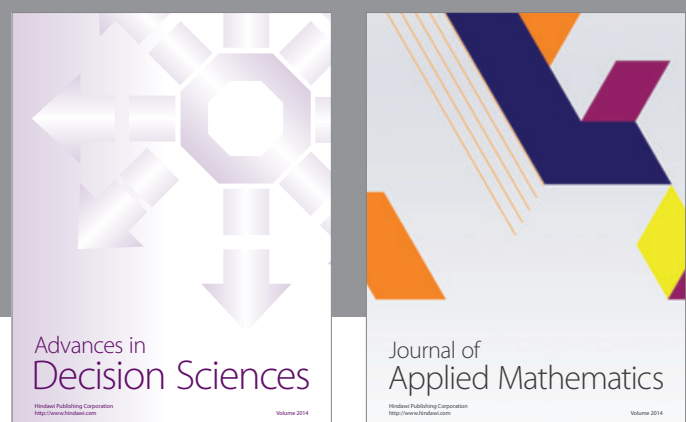

Journal of

Applied Mathematics
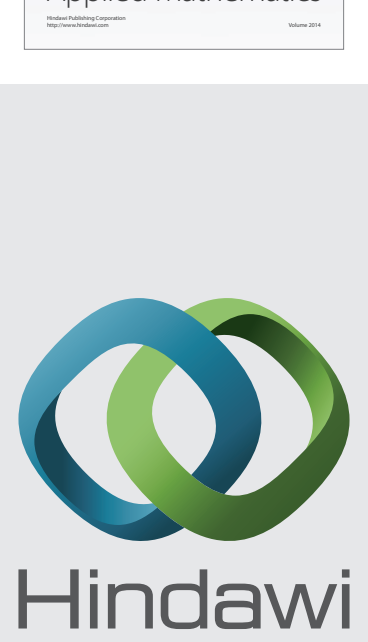

Submit your manuscripts at http://www.hindawi.com
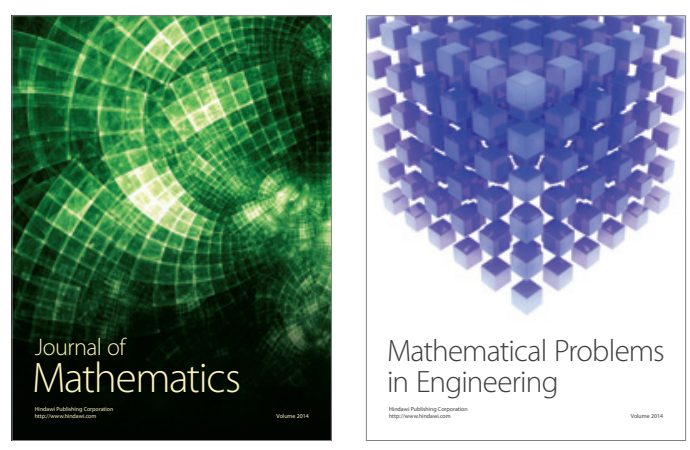

Mathematical Problems in Engineering
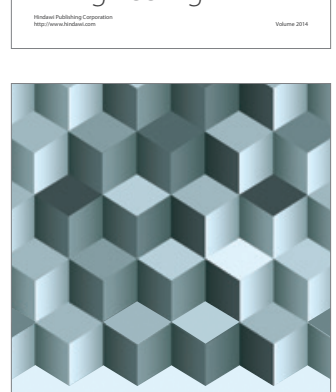

Journal of

Function Spaces
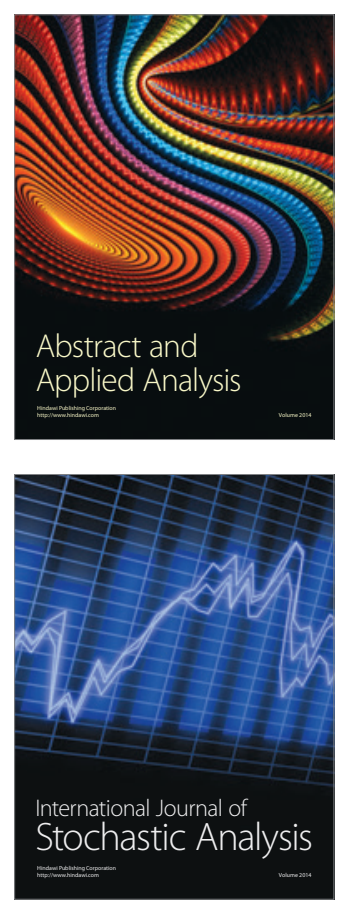

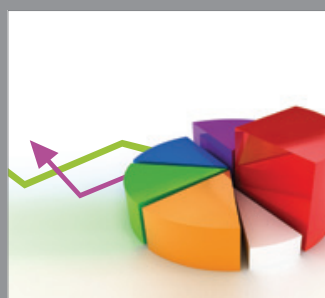

ournal of

Probability and Statistics

Promensencen
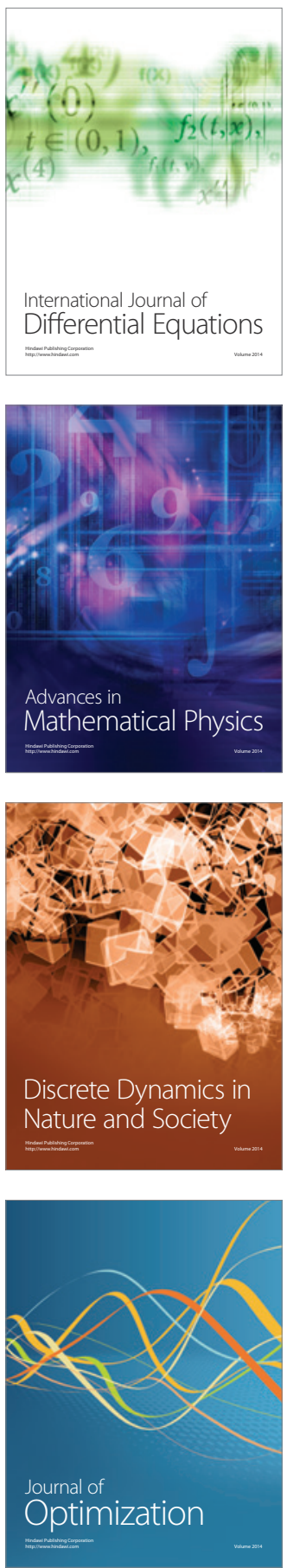